\title{
Comparative vascular anatomies of some orchid species
}

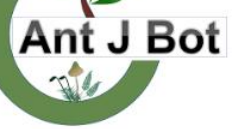

Received : 30.03 .2021

Accepted : 10.06.2021

Online : 22.06 .2021
Şenay SÜNGÜ ŞEKER ${ }^{1} \odot$, Gülcan ŞENEL ${ }^{2 *} \odot$, Mustafa Kemal AKBULUT ${ }^{3} \odot$

${ }^{1,2}$ Ondokuz Mayıs University, Sciences and Arts Faculty, Department of Biology, Samsun, Turkey

${ }^{3}$ Çanakkale Onsekiz Mart University, Lapseki Vocational School, Department of Landscaping and Ornamental

Plants, Çanakkale, Turkey

*gsenel@omu.edu.tr, ${ }^{1}$ senay.sungu@omu.edu.tr, ${ }^{3}$ mkakbulut@comu.edu.tr

\section{Bazı orkide türlerinin karşılaştırmalı damar anatomileri}

Abstract: In this study, we examined the vascular anatomy of leaves with different morphological features of 11 orchid species. Plant samples were collected from various localities in the Black Sea Region. Fresh leaves were dried and stocked, and their vascular structures were analyzed by clearing and staining. Significant differences were determined in the leaves of taxa in terms of characters such as total leaf perimeter and area, number of veins and nodes, total vein length, total vein area, average vein length, average vein width, average vein surface area, average vein volume, and average areolar area. According to the findings, the topological and morphometric features of the veining can reflect the systematic and phylogenetic relationships of orchids.

Key words: Anatomy, venation network, morphometry, Orchidaceae

Özet: $\mathrm{Bu}$ çalışmada 11 orkide türüne ait farklı morfolojik özellikleri olan yaprakların damar anatomileri incelenmiştir. Bitki örnekleri Karadeniz Bölgesi’ndeki çeşitli lokalitelerden toplanmıştır. Taze yapraklar kurutularak stoklanmış, saydamlaştırma ve boyama işlemi uygulanarak damar yapıları analiz edilmiştir. Taksonların yapraklarında toplam yaprak çevresi ve alanı, damar ve boğum sayısı, toplam damar uzunluğu, toplam damar alanı, ortalama damar uzunluğu, ortalama damar genișliği, ortalama damar yüzey alanları, ortalama damar hacmi ve ortalama areol alanı gibi karakterler bakımından önemli farklılıklar tespit edilmiştir. Bulgulara göre damarlanmanın topolojik ve morfometrik özellikleri, orkidelerin sistematik ve filogenetik ilişkilerini yansitabilir.

Anahtar Kelimeler: Anatomi, damar ağı, morfometri, Orchidaceae

Citation: Süngü Şeker Ş, Şenel G, Akbulut MK (2021). Comparative vascular anatomies of some orchid species. Anatolian Journal of Botany 5(2): 84-90.

\section{Introduction}

One of the largest families in the angiosperms of Orchidaceae display natural distribution with over 200 taxa in Turkey (Güner and Aslan, 2012). Additionally, there are many rare and/or endemic orchid species in our country. In addition to consumption of orchids as drinks (sahlep), use in ice cream production and cultivation as decorative plants, they have economic value as an effective treatment for diseases like tuberculosis, chest pain and asthma (Sezik, 1984; Hossain, 2011). However, due to species displaying distribution in a very large area, excessive intraspecies variation of flowers and leaves especially and high hybridization abilities, there are many systematic problems with orchids (Arditti, 1977; Dressler, 1993). Additionally, mistaken plant identification and different taxonomic opinions further complicate these problems.

The leaves of land plants display diversity in terms of vein architecture (Roth-Nebelsick et al., 2001). Monocot plants have main veins organized parallel to the midrib and anastomosis at the distal and basal tips of the leaf blade. Secondary veins have transverse pattern, and develop between main veins to form short interconnections (Conklin et al., 2019). The architecture of the vascular bundles is affected by the phylogenetic or genetic character of the plant in addition to the ecological conditions it exists in (Sack et al., 2012; Blonder et al., 2020). Additionally, led by carbohydrate and watercarrying capacity, they ensure the occurrence of many basic physiological functions like photosynthesis and perspiration, resistance to injury, mechanical support or lignin-derived costs (Vincent, 1982; Niklas, 1999; Givnish et al., 2005; Brobbrib et al., 2007; Agrawal and Konno, 2009; Katifori et al., 2010; Brodribb et al., 2016; John et al., 2017; Blonder et al., 2011, 2018; Ohtsuka et al., 2018). For this reason, the vein shapes represent the basis for studies in many disciplines. However, there are very limited numbers of studies about the vein anatomy and vein patterns in orchids, especially (Cameron and Dickison, 1998; Mani et al., 2021). This research comparatively investigates the vein shapes and structure of 11 genera among representative species included in the Orchidaceae family (Cephalanthera Rich., Coeloglossum Lindl., Dactylorhiza Neck. Ex Nevski, Gymnadenia R.Br., Hymantoglossum Spreng., Limodorum L., Orchis L., Platanthera Rich., Serapias L., Spiranthes Rich., Steveniella Schltr.) distributed in the Black Sea Region to test the usefulness of vein architecture as a characteristic showing systematic relationships between genera. This study was also applied for the first time on the leaf vein structures of orchids in Turkey.

\section{Materials and Method}

Plant samples were collected from a variety of localities in the Black Sea region (Table 1). For each species, mean 10 leaf samples were pressed and dried for several days at 60 ${ }^{\circ} \mathrm{C}$. Leaf samples were taken from at least three individuals. Three different leaves for each individuals were studied, with leaf sizes being small, medium and large. ANOVA test was performed on the obtained data to 
determine the importance of leaf-anatomical data for taxa. Tests were completed with the aid of SPSS 20 program.

\subsection{Clearing}

The transparency method was applied by taking Vasco et al. (2014) as reference. Accordingly, dry leaf samples were left for $1-3$ days at $40-54{ }^{\circ} \mathrm{C}$ in $5 \% \mathrm{NaOH}$ solution.

Samples were washed with distilled water and treated for durations lasting from $20 \mathrm{~s}$ to $10 \mathrm{~min}$ with $4.5-5.5 \%$ sodium hypochlorite. Leaf samples were passed through a graded ethanol series $(50 \%, 70 \%, 95 \%)$ and stained with safranin by applying the standard protocol.

\subsection{Photograph Metadata and Analysis}

Leaves with scale added were photographed from the same distance using a NIKON D700 brand camera and analyzed with the aid of the LEAF GUI (Price et al., 2011) program. After a series of cropping and cleaning procedures, RGB photographs were converted to grayscale and vein, node and areole features were calculated. While determining the threshold value, global and local adaptive thresholding approaches were used to obtain binary images representing vein regions with one and nonvein regions with zero, and vein segmentation was achieved. In the vein network, nodes and vein tips were determined with representative single pixel width. Metric calculations for veins and the full leaf were completed using software.

\section{Results}

When the vein topology of leaves from 11 species belonging to the Orchidaceae family are investigated, the taxa displayed clear differentiation based on secondary vein pattern. In Limodorum abortivum (L.) Sw., the parallel vein pattern disappears at the leaf tips. For Cephalanthera kotchyana Renz \& Taubenheim, Coeloglossum viride Hartm., Dactylorhiza urvilleana (Steud.) H.Baumann \& Künkele, and Gymnadenia conopsea (L.) R.Br. secondary connections between parallel veins are notable (Fig. 1). These connections intensify toward the leaf tip.

Table 1. List of Orchidaceae taxa, localities and collection date

\begin{tabular}{|c|c|c|c|c|c|c|}
\hline Taxa & Subfamily & Locality & Collection date & Voucher & Habitats & Elevation \\
\hline Cephalanthera kotschyana & $\mathrm{E}$ & Kavak, Samsun & April, 2015 & Ss, 29 & Deciduous forests & 750 \\
\hline Cephalanthera kotschyana & $\mathrm{E}$ & Kurupelit, Samsun & April, 2015 & Omuhb, 7711 & Open forests & 225 \\
\hline Coeloglossum viride & $\mathrm{O}$ & Köprübaşı, Trabzon & June, 2015 & Omub, 8253 & Meadows & 1820 \\
\hline Dactylorhiza urvilleana & $\mathrm{O}$ & Köprübaş1, Trabzon & June, 2015 & Omub, 7787 & Open forests, meadows & 850 \\
\hline Dactylorhiza urvilleana & $\mathrm{O}$ & Kavron, Rize & June, 2015 & Omub, 7818 & $\begin{array}{c}\text { Alpine to subalpine } \\
\text { meadows }\end{array}$ & 2000 \\
\hline Dactylorhiza urvilleana & $\mathrm{O}$ & Santa, Gümüşhane & June, 2015 & Omub, 8066 & $\begin{array}{l}\text { Alpine to subalpine } \\
\text { meadows }\end{array}$ & 1600 \\
\hline Gymnadenia conopsea & $\mathrm{O}$ & Köprübaşı, Trabzon & July, 2015 & Mka, 27 & $\begin{array}{c}\text { Alpine to subalpine } \\
\text { meadows }\end{array}$ & 1670 \\
\hline Himantoglossum caprinum & $\mathrm{O}$ & Kurupelit, Samsun & June, 2015 & Omuhb, 7739 & $\begin{array}{l}\text { Coniferous forests, open } \\
\text { forests }\end{array}$ & 245 \\
\hline Himantoglossum caprinum & $\mathrm{O}$ & Boyabat, Sinop & June, 2015 & Mka, 28 & Coniferous forests, & 300 \\
\hline Limodorum abortivum & $\mathrm{E}$ & Kavak, Samsun & June, 2015 & Mka, 30 & Deciduous forests & 450 \\
\hline Limodorum abortivum & $\mathrm{E}$ & Kurupelit, Samsun & June, 2015 & Omuhb, 3036 & Quercus forests & 150 \\
\hline Orchis mascula subsp. pinetorum & $\mathrm{O}$ & Abant, Bolu & May, 2015 & Ss, 16 & Edges of coniferous forests & 985 \\
\hline Orchis mascula subsp. pinetorum & $\mathrm{O}$ & Çambaşı, Ordu & May, 2015 & Omuhb, 7829 & Edges of coniferous forests & 1515 \\
\hline Orchis mascula subsp. pinetorum & $\mathrm{O}$ & Kurupelit, Samsun & May, 2015 & Omuhb, 7712 & Quercus forests & 163 \\
\hline Orchis mascula subsp. pinetorum & $\mathrm{O}$ & Köprübaşı, Trabzon & June, 2015 & Omuhb, 7725 & Open areas, meadows & 2300 \\
\hline Platanthera chlorantha & $\mathrm{O}$ & Kavak, Samsun & June, 2015 & Mka, 33 & Deciduous forests & 870 \\
\hline Platanthera chlorantha & $\mathrm{O}$ & Kurupelit, Samsun & June, 2015 & Omuhb, 4123 & $\begin{array}{l}\text { Deciduous forests, } \\
\text { Quercus forests }\end{array}$ & 157 \\
\hline Serapias bergonii & $\mathrm{O}$ & Ünye, Ordu & May, 2015 & Mka, 36 & Meadows & 60 \\
\hline Serapias bergonii & $\mathrm{O}$ & Kale, Giresun & May, 2015 & Mka, 34 & Meadows & 90 \\
\hline Spiranthes spiralis & $\mathrm{O}$ & Köprübaş1, Trabzon & Sep, 2015 & Mka, 49 & Open forests, meadows & 860 \\
\hline Spiranthes spiralis & $\mathrm{O}$ & Kurupelit, Samsun & Sep, 2015 & Mka, 38 & Meadows & 205 \\
\hline Steveniella satyrioides & $\mathrm{O}$ & Ünye, Ordu & May, 2015 & Mka, 50 & Edges of deciduous forests & 90 \\
\hline Steveniella satyrioides & $\mathrm{O}$ & Bafra, Samsun & May, 2015 & Mka, 40 & Edges of deciduous forests & 120 \\
\hline Steveniella satyrioides & $\mathrm{O}$ & Kurupelit, Samsun & May, 2015 & Omuhb, 3041 & $\begin{array}{l}\text { Deciduous forests, } \\
\text { Quercus forests }\end{array}$ & 210 \\
\hline
\end{tabular}

E: Epidendroideae, O: Orchidoideae 
In Orchis mascula subsp. pinetorum (Boiss. \& Kotschy) G.Camus, Serapias bergonii E.G.Camus and Steveniella satryoides (Spreng,) Schltr the secondary vein network is distributed homogeneously throughout the full leaf blade, different to other species. In Himantoglossum caprinum (M.Bieb.) Spreng and Platanthera clorantha (Cruster) Rchb. the secondary vein network between the primary parallel veins displays net-like pattern. Additionally, the free tipped secondary veins are distributed between areoles in these taxa. In Spiranthes spiralis (L.) Chevall, the secondary veins have wave-like pattern and as a result of this pattern, Spiranthe spiralis has irregular areoles compared to the rectangular shape of areoles in other species (Fig. 1).

For vein morphometric properties, metric features like total leaf circumference and area; total vein network length and area; vein and node numbers; mean vein length, width, 2D, 3D area and volume; and mean areole equivalent diameter, circumference, area and convex area were investigated. Leaves from photosynthetic or saprophyte species cultivated in different habitats were identified to have significant differences in terms of general leaf properties, vein and areole features. Total leaf circumference and area, vein and node numbers, total vein network length and total area covered by the vein network were higher for Dactylorhiza urvilleana, Himantoglossum caprinum and Platanthera chlorantha compared to other species. In terms of total leaf features, the lowest values were for Coeloglossum viride and S. spiralis, while lowest vein and node numbers were observed in Cephalanthera kotchyana and Limodorum abortivum (Table 2).

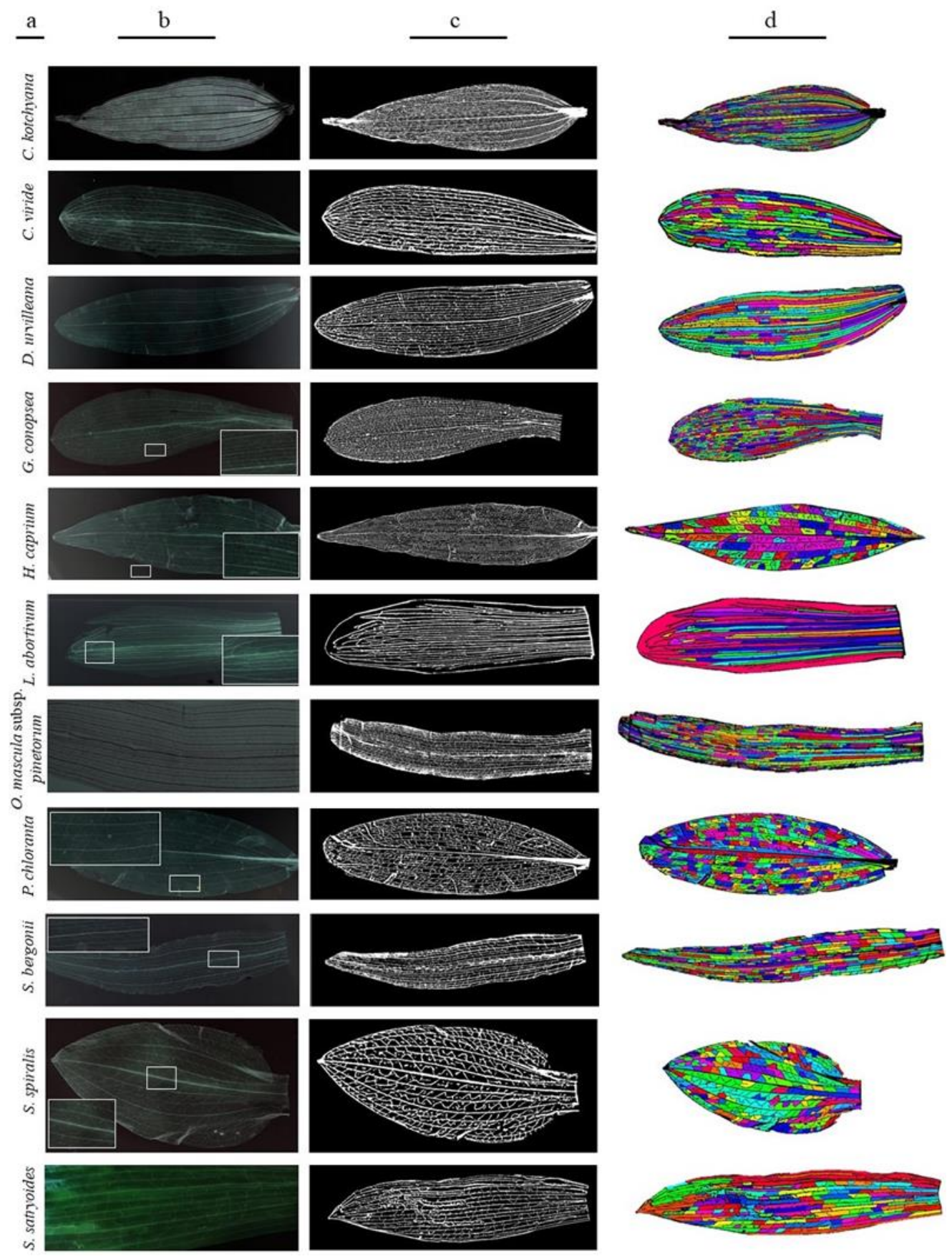

Figure 1. Vein segmentation of taxa. a: taxon b: RGB photographic samples, c: binary images showing veins white and non-vein regions in black, d: image of color labeled areoles (each color represents different areoles) 
Table 2. Average morphometric data of the leaf vein characters of taxa

\begin{tabular}{|c|c|c|c|c|c|c|c|c|c|c|c|c|c|c|c|c|}
\hline \multirow[b]{2}{*}{ 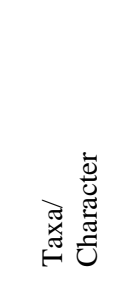 } & \multicolumn{6}{|c|}{ Leaf } & \multicolumn{5}{|c|}{ Vein } & \multicolumn{5}{|c|}{ Areole } \\
\hline & 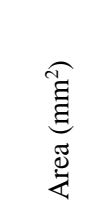 & 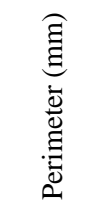 & 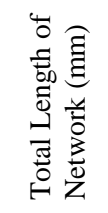 & 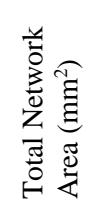 & 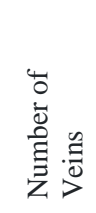 & 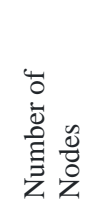 & 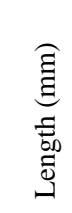 & $\begin{array}{l}\widehat{\Xi} \\
\text { ह્छ } \\
\text { 吾 } \\
\vdots\end{array}$ & 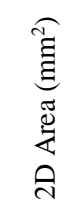 & 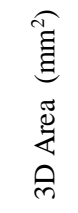 & 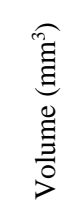 & 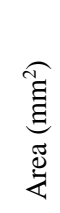 & 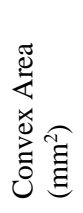 & 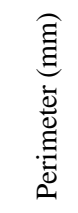 & 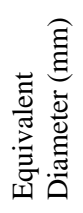 & 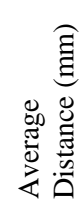 \\
\hline Cep kot & 560.0 & 146.1 & 2159.1 & 210.0 & 1241 & & 1.69 & & 000 & & 00 & & 7.3 & 17.0 & 0.85 & 0.04 \\
\hline & & & 1009.0 & 110.9 & & & & & & & & & & 2.9 & 0.37 & 0.04 \\
\hline Dac urv & 2867.4 & 309.1 & 5230.9 & 869.0 & 14033 & & 0.29 & & 0.03 & 0.09 & 0.01 & 1.5 & 3.0 & 5.9 & 0.49 & 0.05 \\
\hline Gim con & 795.7 & 187.2 & 2779.5 & 280.4 & 7792 & 978 & 0.30 & 0.05 & 0.02 & 0.05 & 0.00 & 0.4 & 0.6 & 3.7 & 0.53 & 0.05 \\
\hline Him cap & 2390.1 & 256.5 & 5682.6 & 717.3 & 18005 & 17926 & 0.23 & 0.06 & 0.02 & 0.05 & 0.00 & 1.0 & 2.2 & 3.2 & 0.20 & 0.03 \\
\hline Lim abo & 599.7 & 110.7 & 1166.1 & 178.7 & 2133 & & 0.48 & & 0.04 & 0.13 & 0.01 & 1.8 & 4.1 & 8.4 & 0.56 & 0.04 \\
\hline Orc mas & 782.8 & 234.9 & 2699.3 & 307.6 & 6857 & 5842 & 0.33 & & 0.02 & & & 0.4 & 0.5 & 3.0 & 0.40 & 0.04 \\
\hline Pla chl & 2562.8 & 232.5 & 5200.6 & 783.9 & 16740 & 15361 & 0.23 & 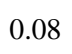 & 0.02 & 0.07 & 0.01 & 1.1 & 1.4 & 4.6 & 0.63 & 0.04 \\
\hline Ser ber & 351.8 & 130.9 & 1106.7 & 115.3 & 2417 & 2140 & 0.39 & 0.06 & 0.02 & 0.07 & 0.00 & 0.4 & 0.6 & 3.3 & 0.47 & 0.07 \\
\hline Spi spi & 327.4 & 87.3 & 1201.0 & 100.9 & 5370 & 4735 & 0.15 & 0.05 & 0.01 & 0.02 & 0.00 & 0.2 & 0.3 & 1.8 & 0.28 & 0.05 \\
\hline Ste sat & 842.2 & 166.7 & 1818.8 & 231.4 & 4454 & 4077 & 0.34 & 0.06 & 0.02 & 0.08 & 0.00 & 1.1 & 1.7 & 5.4 & 0.63 & 0.04 \\
\hline
\end{tabular}

The situation is different for features like mean vein dimensions, vein surface area and volume. Cephalanthera kotchyana and Limodorum abortivum had much higher vein length values compared to other species. Additionally, these species had higher values for 2D and $3 \mathrm{D}$ vein area compared to other species. Spiranthes spiralis had the lowest values for mean vein dimensions (Table 2,3).

For areole features, clear differences were not observed for criteria like the mean distance (mean Euclidean distance between each areole pixel and vein pixel) and equivalent diameter (diameter of the region with the same normalized secondary axis moment). However, there were variability for criteria like areole area, areole convex area (area of the convex shell covering the areole) and areole circumference in many species. These values were high for species like Cephalanthera kotchyana and Limodorum abortivum and lowest for Coeloglossum viride and Spiranthes spiralis (Fig. 2, Table 2).

Table 3. Descriptive statistic for vein and areole of taxa

\begin{tabular}{|c|c|c|c|c|c|c|c|c|c|c|c|}
\hline \multirow[b]{2}{*}{ Taxa } & & \multicolumn{5}{|c|}{ Vein } & \multicolumn{5}{|c|}{ Areole } \\
\hline & & $\begin{array}{l}\text { Length } \\
(\mathrm{mm})\end{array}$ & $\begin{array}{l}\text { Width } \\
(\mathrm{mm})\end{array}$ & $\begin{array}{l}\text { 2D Area } \\
\left(\mathrm{mm}^{2}\right)\end{array}$ & $\begin{array}{l}\text { 3D Area } \\
\left(\mathrm{mm}^{2}\right)\end{array}$ & $\begin{array}{l}\text { Volume } \\
\left(\mathrm{mm}^{3}\right)\end{array}$ & $\begin{array}{c}\text { Area } \\
\left(\mathrm{mm}^{2}\right)\end{array}$ & $\begin{array}{c}\text { Convex } \\
\text { Area } \\
\left(\mathrm{mm}^{2}\right)\end{array}$ & $\begin{array}{c}\text { Perimete } \\
\mathrm{r}(\mathrm{mm})\end{array}$ & $\begin{array}{c}\text { Equivale } \\
\text { nt } \\
\text { Diamete } \\
\text { r (mm) }\end{array}$ & $\begin{array}{c}\text { Average } \\
\text { Distance } \\
(\mathrm{mm})\end{array}$ \\
\hline \multirow{2}{*}{ Cep kot } & Mean & 1.686 & 0.060 & 0.092 & 0.288 & 0.011 & 1.848 & 7.294 & 17.050 & 0.846 & 0.039 \\
\hline & Std. Deviation & 4.180 & 0.063 & 0.199 & 0.625 & 0.029 & 5.001 & 21.300 & 39.912 & 1.283 & 0.014 \\
\hline \multirow{2}{*}{ Coe vir } & Mean & 0.234 & 0.054 & 0.015 & 0.047 & 0.002 & 0.310 & 0.451 & 2.942 & 0.372 & 0.041 \\
\hline & Std. Deviation & 0.368 & 0.029 & 0.031 & 0.098 & 0.006 & 0.698 & 1.165 & 5.828 & 0.506 & 0.024 \\
\hline \multirow{2}{*}{ Dac urv } & Mean & 0.286 & 0.082 & 0.029 & 0.091 & 0.006 & 1.488 & 2.985 & 5.934 & 0.490 & 0.048 \\
\hline & Std. Deviation & 0.550 & 0.055 & 0.080 & 0.253 & 0.036 & 7.974 & 22.780 & 26.640 & 1.287 & 0.043 \\
\hline \multirow{2}{*}{ Gim con } & Mean & 0.298 & 0.051 & 0.017 & 0.053 & 0.002 & 0.412 & 0.583 & 3.667 & 0.529 & 0.055 \\
\hline & Std. Deviation & 0.430 & 0.022 & 0.031 & 0.098 & 0.005 & 1.071 & 2.067 & 7.697 & 0.495 & 0.025 \\
\hline \multirow{2}{*}{ Him cap } & Mean & 0.232 & 0.060 & 0.017 & 0.055 & 0.003 & 1.010 & 2.249 & 3.196 & 0.204 & 0.034 \\
\hline & Std. Deviation & 0.407 & 0.033 & 0.057 & 0.180 & 0.046 & 12.545 & 30.203 & 35.144 & 1.116 & 0.015 \\
\hline \multirow{2}{*}{ Lim abo } & Mean & 0.478 & 0.066 & 0.041 & 0.128 & 0.006 & 1.763 & 4.125 & 8.369 & 0.558 & 0.042 \\
\hline & Std. Deviation & 1.066 & 0.035 & 0.103 & 0.323 & 0.018 & 11.368 & 37.811 & 41.429 & 1.393 & 0.037 \\
\hline \multirow{2}{*}{ Orc mas } & Mean & 0.327 & 0.066 & 0.022 & 0.068 & 0.003 & 9.579 & 10.085 & 6.310 & 0.482 & 0.041 \\
\hline & Std. Deviation & 0.499 & 0.042 & 0.033 & 0.103 & 0.006 & 77.204 & 81.280 & 49.989 & 3.486 & 0.114 \\
\hline \multirow{2}{*}{ Pla chl } & Mean & 0.227 & 0.081 & 0.022 & 0.069 & 0.006 & 1.077 & 1.438 & 4.595 & 0.628 & 0.070 \\
\hline & Std. Deviation & 0.278 & 0.059 & 0.058 & 0.182 & 0.070 & 3.547 & 5.437 & 12.131 & 0.989 & 0.064 \\
\hline \multirow{2}{*}{ Ser ber } & Mean & 0.388 & 0.060 & 0.023 & 0.072 & 0.003 & 0.393 & 0.594 & 3.283 & 0.471 & 0.052 \\
\hline & Std. Deviation & 0.617 & 0.034 & 0.033 & 0.104 & 0.004 & 1.317 & 3.302 & 9.572 & 0.528 & 0.028 \\
\hline \multirow{2}{*}{ Spi spi } & Mean & 0.154 & 0.047 & 0.008 & 0.025 & 0.001 & 0.218 & 0.297 & 1.787 & 0.277 & 0.040 \\
\hline & Std. Deviation & 0.170 & 0.024 & 0.012 & 0.037 & 0.002 & 0.766 & 1.068 & 4.585 & 0.449 & 0.030 \\
\hline \multirow{2}{*}{ Ste sat } & Mean & 1.686 & 0.060 & 0.092 & 0.288 & 0.011 & 1.114 & 1.666 & 5.406 & 0.634 & 0.063 \\
\hline & Std. Deviation & 4.180 & 0.063 & 0.199 & 0.625 & 0.029 & 3.287 & 6.403 & 16.438 & 1.010 & 0.058 \\
\hline
\end{tabular}



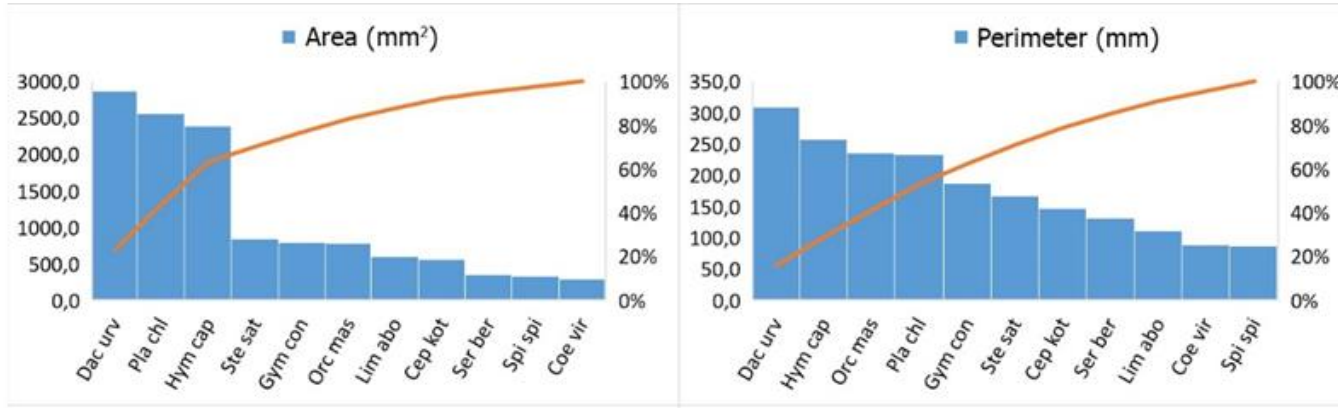

- Total Length of Network (mm)

= Total Network Area $\left(\mathrm{mm}^{2}\right)$
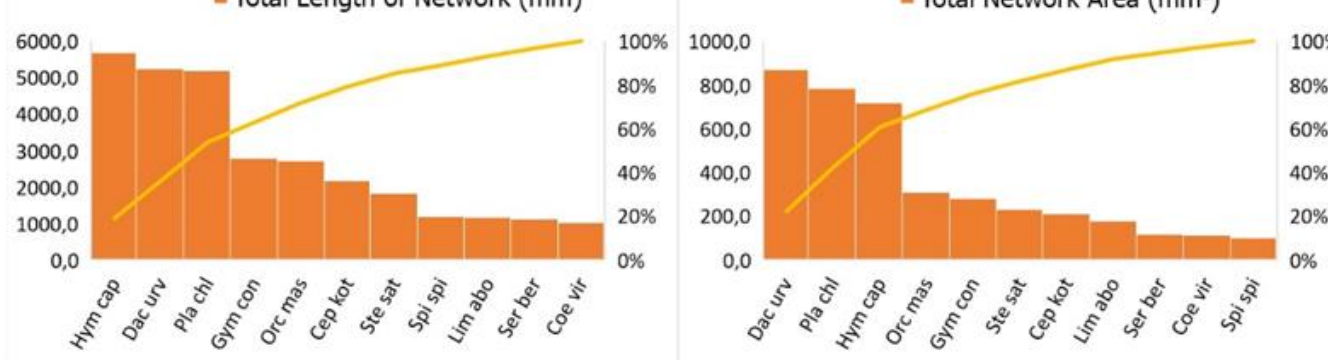

= Number of Veins

n Number of Nodes
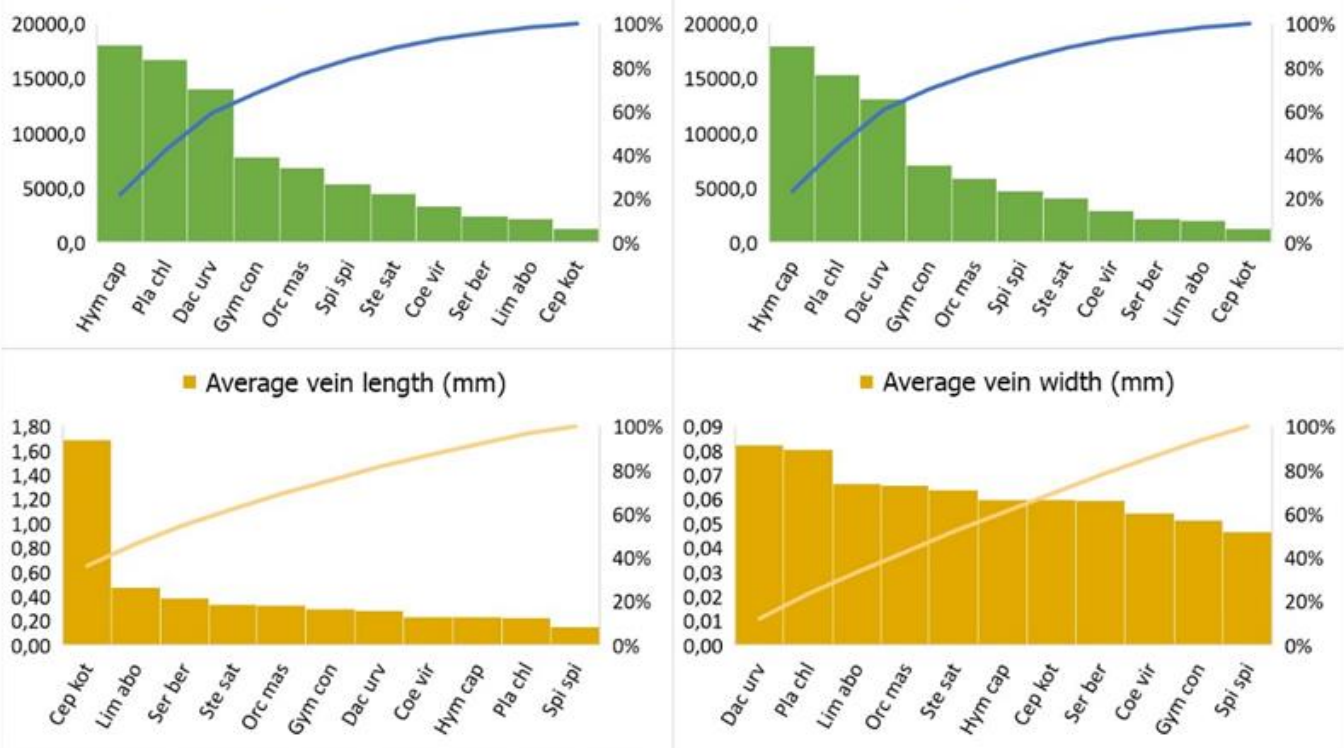

- Average $2 \mathrm{D}$ Area $\left(\mathrm{mm}^{2}\right)$

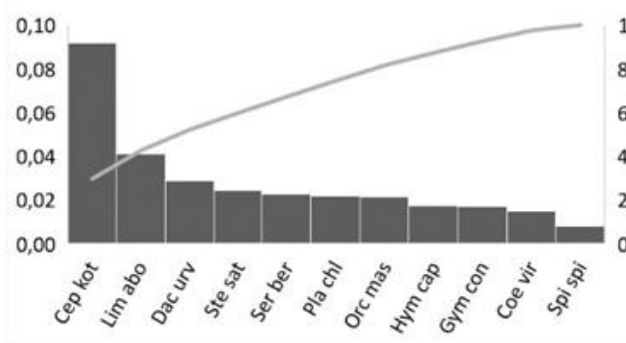

- Average 3D Area $\left(\mathrm{mm}^{2}\right)$

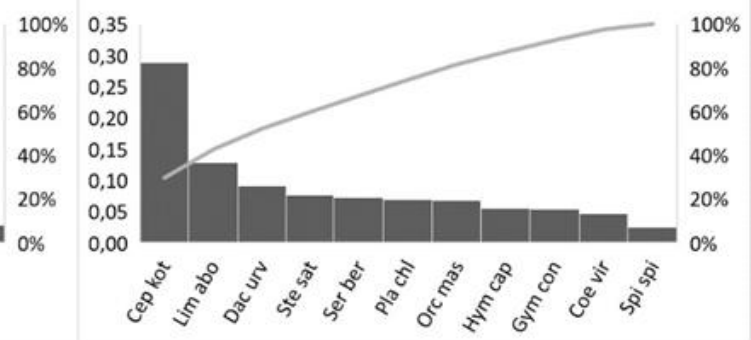

" Average volume $\left(\mathrm{mm}^{3}\right)$

" Avarege areole area $\left(\mathrm{mm}^{2}\right)$

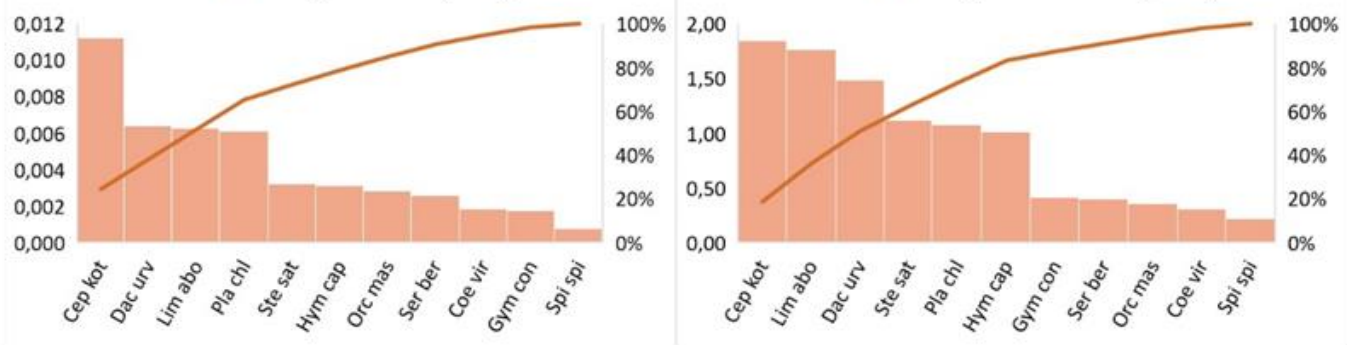

Figure 2. Pareto graphs of vein morphometric data 
It was revealed that the characters using ANOVA test were important for at least one taxa (Table 4).

\section{Discussions}

Orchidaceae is taxonomically divided into five subfamilies (Epidendroideae, Orchidoideae, Vanilloideae, Cyprepedioideae and Apostasioideae). The species common in Turkey are from the Epidendroideae and Orchidoideae subfamilies. The members of this family are common in the whole country (Sezik, 1984). The leaf vein topographic and morphometric features of some representative orchid species growing in Turkey were comparatively assessed between genera. These features clearly separated the epidendroid and orchidoid taxa. Software defined the junction point of two or more vein elements as a node and defined the vein segments between the two nodes as veins (Larese et al., 2014). The vein and node numbers in epidendroid species were fewer than for orchidoid species. In other words, sparse location of secondary connecting veins between parallel veins causes higher mean vein and areole dimensions. For this reason, these characteristics reflect the phylogenetic differentiation between two subfamilies (epidendroid and orchidoid) of the investigated species (Cameron et al., 1999).

Among Orchideae tribes members which were the topic of the study, Spiranthes spiralis was classified within the Cranichideae tribes, different to other orchidoid species. This is different to other orchidoid taxa due to leaves characterized by wavy secondary veins and irregular areole shapes. In this group, Coeloglossum viride, Dactylorhiza urvilleana, and Gymnadenia conopsea taxa are closely related on dendrograms obtained with molecular markers (Pridgeon, 1997; Batemann et al., 2003). In these three taxa, secondary vein connections intensify toward leaf tips, and the topology of these veins may indicate characteristics shared at the genera level.

Table 4. ANOVA analysis for vein and areole characters

\begin{tabular}{|c|c|c|c|c|c|c|c|}
\hline & Charachters & & Sum of Squares & df & Mean Square & $\mathbf{F}$ & Sig. \\
\hline \multirow{5}{*}{$\frac{\Xi}{2}$} & Length & \multirow{10}{*}{ 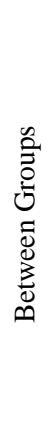 } & 2967.46 & 10.00 & 269.77 & 534.40 & 0.00 \\
\hline & Width & & 12.24 & 10.00 & 1.11 & 580.45 & 0.00 \\
\hline & 2D Area & & 10.82 & 10.00 & 0.98 & 244.20 & 0.00 \\
\hline & 3D Area & & 106.76 & 10.00 & 9.71 & 244.20 & 0.00 \\
\hline & Volume & & 0.40 & 10.00 & 0.04 & 20.94 & 0.00 \\
\hline \multirow{5}{*}{$\begin{array}{l}\frac{0}{0} \\
\frac{0}{2} \\
\stackrel{4}{4}\end{array}$} & Area & & 7098.31 & 10.00 & 645.30 & 8.10 & 0.00 \\
\hline & Convex Area & & 19606.59 & 10.00 & 1782.42 & 5.84 & 0.00 \\
\hline & Perimeter & & 44539.87 & 10.00 & 4049.08 & 9.39 & 0.00 \\
\hline & Equivalent Diameter & & 229.52 & 10.00 & 20.87 & 23.38 & 0.00 \\
\hline & Average Distance & & 1.36 & 10.00 & 0.12 & 79.67 & 0.00 \\
\hline
\end{tabular}

Many species may be characterized in terms of criteria evaluating vein and areole structure. The results show that the topology of secondary veins between parallel veins in orchids are preserved during evolution. For this reason, it is considered that when preliminary preparation procedures are completed correctly and carefully, this method will provide easy, rapid and reliable data which will be beneficial to assess relationships between genera in problematic groups like orchids or to identify possible intergeneric hybrid taxa.

\section{Conflict of Interest}

Authors have declared no conflict of interest.

\section{Authors' Contributions}

The authors contributed equally.

\section{Acknowledgments}

This research was supported by the Scientific and Technological Research Council of Turkey (114Z702).

\section{References}

Agrawal AA, Konno K (2009). Latex: A model for understanding mechanisms, ecology, and evolution of plant defense against herbivory. Annual Review of Ecology Evolution and Systematics 40:311-331.

Arditti J (1977). Orchid biology, reviews and perspectives. Ithaca: Cornel Univ. Press.

Bateman RM, Hollingsworth PM, Preston J, Yi-Bo L, Pridgeon AM, Chase MW (2003). Molecular phylogenetics and evolution of Orchidinae and selected Habenariinae (Orchidaceae). Botanical journal of the Linnean Society 142(1): 1-40.

Blonder B, Both S, Jodra M, Xu H, Fricker M, Matos IS, Majalap N, Burslem DFRP, Teh YA, Malhi Y (2020). Linking functional traits to multiscale statistics of leaf venation networks. New Phytologist 228: 1796-1810.

Blonder B, Salinas N, Bentley LP, Shenkin A, Chambi Porroa PO, Valdez Tejeira Y, Asner GP (2018). Structural and defensive roles of angiosperm leaf venation network reticulation across an Andes-Amazon Elevation Gradient. Journal of Ecology 106(4): 1683-1699.

Blonder B, Violle C, Bentley LP, Enquist BJ (2011). Venation networks and the origin of the leaf economics spectrum. Ecology letters 14(2): 91-100.

Brodribb TJ, Bienaimé D, Marmottant P. (2016). Revealing catastrophic failure of leaf networks under stress. Proceedings of the National Academy of Sciences 113(17): 4865-4869. 
Brodribb TJ, Feild TS, Jordan GJ (2007). Leaf maximum photosynthetic rate and venation are linked by hydraulics. Plant Physiology 144(4): 1890-1898.

Cameron KM, Dickison WC (1998). Foliar architecture of vanilloid orchids: insights into the evolution of reticulate leaf venation in monocotyledons. Botanical Journal of the Linnean Society 128(1): 45-70.

Cameron KM, Chase MW, Whitten WM, Kores PJ, Jarrell DC, Albert VA, Goldman DH (1999). A phylogenetic analysis of the Orchidaceae: evidence from rbcL nucleotide sequences. American Journal of Botany 86(2): 208-224.

Conklin PA, Strable J, Li S, Scanlon MJ (2019). On the mechanisms of development in monocot and eudicot leaves. New Phytologist 221(2): 706-724.

Dressler RL (1993). Phylogeny and classification of the orchid family. Oregon: Dioscorides Press.

Givnish TJ, Pires JC, Graham SW, McPherson MA, Prince LM, Patterson TB, Millam KC (2005). Repeated evolution of net venation and fleshy fruits among monocots in shaded habitats confirms a priori predictions: Evidence from an $\mathrm{ndhF}$ phylogeny. Proceedings of the Royal Society B: Biological Sciences 272(1571): 1481-1490.

Güner A, Aslan S (Eds.) (2012). Türkiye bitkileri listesi:(damarlı bitkiler). İstanbul: Nezahat Gökyiğit Botanik Bahçesi Yayınları.

Hossain MM (2011). Therapeutic orchids: traditional uses and recent advances an overview. Fitoterapia 82(2): 102-140.

John GP, Scoffoni C, Buckley TN, Villar R, Poorter H, Sack L (2017). The anatomical and compositional basis of leaf mass per area. Ecology Letters 20(4): 412-425.

Katifori E, Szöllősi GJ, Magnasco MO (2010). Damage and fluctuations induce loops in optimal transport networks. Physical Review Letters 104(4): 048704

Larese MG, Namías R, Craviotto RM, Arango MR, Gallo C, Granitto PM (2014). Automatic classification of legumes using leaf vein image features. Pattern Recognition 47(1): 158-168.

Mani M, Rasangam L, Selvam P, Shekhawat MS (2021). Micro-morpho-anatomical mechanisms involve in epiphytic adaptation of micropropagated plants of Vanda tessellata (Roxb.) Hook. ex G. Don. Microscopy Research and Technique 84: 712722.

Niklas KJ (1999). A mechanical perspective on foliage leaf form and function. The New Phytologist 143(1): 19-31.

Ohtsuka A, Sack L, Taneda H (2018). Bundle sheath lignification mediates the linkage of leaf hydraulics and venation. Plant, Cell and Environment 41(2): 342-353.

Price CA, Symonova O, Mileyko Y, Hilley T, Weitz JS (2011). Leaf extraction and analysis framework graphical user interface: Segmenting and analyzing the structure of leaf veins and areoles. Plant Physiology 155(1): 236-245.

Pridgeon AM (1997). Phylogenetics of subtribe Orchidinae (Orchidoideae, Orchidaceae) based on nuclear ITS sequences. 1. Intergeneric relationships and polyphyly of Orchis sensu lato. Lindleyana 12: 89-109.

Roth-Nebelsick A, Uhl D, Mosbrugger V, Kerp H (2001). Evolution and function of leaf venation architecture: a review. Annals of Botany 87(5): 553-566.

Sack L, Scoffoni C, McKown AD, Frole K, Rawls M, Havran JC, Tran T (2012). Developmentally based scaling of leaf venation architecture explains global ecological patterns. Nature Communications 3(1): 1-10.

Sezik EE (1984). Orkidelerimiz: Türkiye'nin orkideleri. İstanbul: Sandoz Kültür Yayınları.

Vasco A, Thadeo M, Conover M, Daly DC (2014). Preparation of Samples for Leaf Architecture Studies, a Method for Mounting Cleared Leaves. Applications in Plant Sciences 2(9): 1400038.

Vincent JF (1982). The mechanical design of grass. Journal of Materials Science 17(3): 856-860. 